\title{
An unusual cause of peripheral neuropathy
}

\author{
Syed Zaman, Andrew Latchford, Stephen Allen
}

A 74-year-old man presented with a 2-month history of weight gain, lethargy and cold intolerance. He had had endoscopic retrograde cholangiopancreatography (ERCP) 3 years prior to admission. His free thyroxine was $<5 \mathrm{pmol} / \mathrm{l}$, and thyroid-stimulating hormone was $>100 \mathrm{mU} / 1$. Thyroxine replacement therapy was commenced. On review 4 weeks later he complained of progressive leg weakness; he denied back pain, sphincter disturbance or sensory symptoms.

There were bilateral lower motor neuron signs in his legs with global weakness, grade $3 / 5$. Sensory testing showed some reduction in all modalities in the lower limbs and his upper limbs were normal. Investigations showed a normal full blood count, erythrocyte sedimentation rate (ESR) was $38 \mathrm{~mm} / \mathrm{h}$, renal and liver function tests were normal as were serum calcium, glucose, vitamin $\mathrm{B} 12$ and folate; the autoimmune profile was negative and protein electrophoresis showed an acute phase pattern; chest X-ray and magnetic resonance imaging of the lumbar spine were normal, while electromyography showed severe neurogenic changes in the lower limb muscles consistent with a radiculopathy and absent sensory action potentials; cerebrospinal fluid (CSF) protein was $0.53 \mathrm{~g} / 1$ with no pleocytosis.

Out-patient physiotherapy was arranged. He was subsequently re-admitted with fever and rigors. Apart from a temperature of $38.1^{\circ} \mathrm{C}$, general examination was unremarkable and his neurological signs were unchanged. Further investigations showed: ESR $68 \mathrm{~mm} / \mathrm{h}$, C-reactive protein $159 \mathrm{mg} / \mathrm{l}$, white blood cell count $7 \times 10^{9} / 1$ (neutrophils $\left.5.4 \times 10^{9} / 1\right)$, alanine transaminase $105 \mathrm{IU} / \mathrm{l}$, bilirubin $30 \mu \mathrm{M} / 1$, alkaline phosphatase $164 \mathrm{IU} / 1$; blood and urine cultures were sterile. Liver ultrasound was performed (figure).

\author{
Department of \\ Medicine, The Royal \\ Bournemouth \\ Hospital, \\ Bournemouth, Dorset \\ BH7 7DW, UK \\ $S$ Zaman \\ A Latchford \\ $\mathrm{S}$ Allen
}

Accepted 19 November 1997

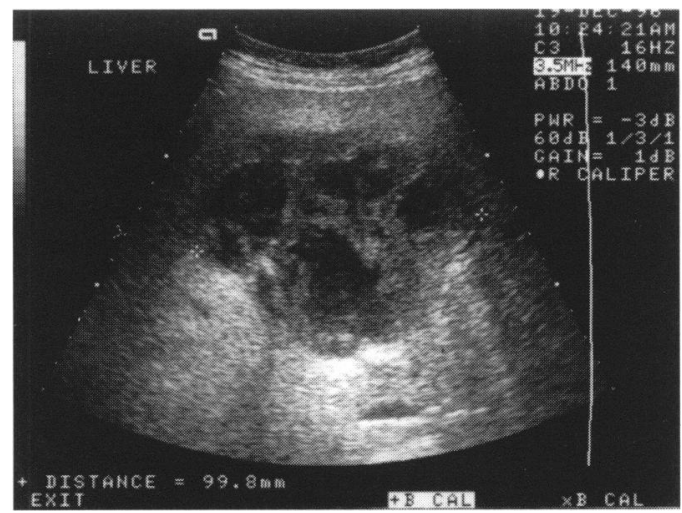

\section{Questions}

1 What does the ultrasound show and, given the information provided, what is the most likely diagnosis?

2 How does this condition usually present?

3 What is the treatment? 


\section{Answers}

QUESTION 1

There is a thick-walled, part cystic, part solid complex mass of approximately $10 \mathrm{~cm}$ lying within the liver. The presence of fever and rigors, elevated inflammatory markers and abnormal liver function tests makes a pyogenic liver abscess likely although a necrotic metastasis is another possibility. Percutaneous aspiration biopsy confirmed the former diagnosis although no organism was isolated on culture.

\section{QUESTION 2}

The usual modes of presentation of an hepatic abscess are listed in box 1 . Abscesses usually present with local symptoms and signs often accompanied by evidence of systemic infection, eg, fever, tachycardia and hypotension. The site of abdominal pain is usually the right upper quadrant but it can be generalised. Nonspecific presentation is common in the elderly making early diagnosis difficult.

\section{QUESTION 3}

The diagnosis should be confirmed by ultrasound-guided aspiration biopsy. Percutaneous catheter drainage should be performed, combined with appropriate antibiotics. However, initial antibiotic therapy should be started blind, bearing in mind the most likely pathogens: $E$ coli and other Gram-negative rods, Staphylococcus aureus, anaerobes and streptococcal species. Antibiotics should be continued for at least 2 weeks or until closure of the abscess cavity has been confirmed by ultrasound. Laparotomy with open surgical drainage is indicated for failed percutaneous drainage.

Our patient was started on intravenous cefotaxime and metronidazole; his fever resolved and his inflammatory markers and liver function tests normalised. Lower limb power and sensation gradually returned to normal over a 4-month period at which point repeat liver ultrasound confirmed complete resolution of the abscess and antibiotics were stopped.

\begin{tabular}{|l|}
\hline Usual modes of presentation of an \\
hepatic abscess \\
\hline Symptoms \\
- fever \\
- rigors \\
- malaise \\
- weight loss \\
- nausea and vomiting \\
Signs \\
- jaundice \\
- pyrexia \\
- tachycardia \\
- hepatomegaly \\
- rachypnoea \\
- ascites pleural effusion/rub \\
- splenomegaly \\
- delirium \\
- clubbing \\
\hline
\end{tabular}

Box 1

\section{Causes of peripheral neuropathy}

- idiopathic chronic sensorimotor

- post-infective (Guillain-Barre syndrome)

- drugs/toxins

- metabolic (diabetes mellitus, hypothyroidism)

- vitamin deficiency (B12)

- neoplastic disease

- connective tissue disease

- hereditary sensorimotor

Box 2

\section{Discussion}

The patient was grossly hypothyroid at presentation. This could explain the sensorimotor neuropathy but it was unusual that he should develop limb weakness after starting thyroxine. Other causes of peripheral neuropathy (box 2) were excluded. Since the leg weakness improved with antibiotic treatment, we propose that the underlying suppurative illness was responsible for the neuromuscular abnormalities. But by what mechanism? The systemic inflammatory response syndrome (SIRS) is due to systemic activation of cytokines in response to sepsis, trauma and burns. Critical illness polyneuropathy is one manifestation of SIRS; it is clinically and electrophysiologically distinct from the Guillain-Barre syndrome ${ }^{1}$ and results from microcirculatory changes to peripheral nerves. It usually presents with difficulty weaning from a ventilator and limb weakness. Histologically there is evidence of neuropathy and myopathy with axonal degeneration of peripheral sensory and motor nerve fibres and muscle fibre atrophy with occasional necrosis. Perhaps our patient had a subacute or chronic form of this disorder. It should be emphasised that pyogenic liver abscess is an uncommon disorder with a high mortality rate but it does have a predilection for the elderly. There is an association with cholelithiasis, diverticulitis and biliary tract surgery but spontaneous abscess formation has also been described. ${ }^{2}$ Our patients' ERCP, though years before presentation, may have been the portal of entry. In a review of 17 elderly patients with liver abscesses ${ }^{3}$ and another review of 26 patients, ${ }^{4}$ presentation with peripheral neuropathy is not described. Hepatic abscess may also be the initial manifesta tion of colonic carcinoma ${ }^{5}$; our patients' barium enema with computed tomography showed no evidence of colonic disease.

\section{Summary points}

- weak legs due to myopathy/neuropathy can occur as a remote effect of pyogenic infection

- the aetiology is unclear but may be due to the systemic inflammatory response syndrome

- the neuromuscular dysfunction is reversible with appropriate antibiotic treatment 


\section{Final diagnosis}

Peripheral neuropathy secondary to pyogenic liver abscess.

1 Bolton CF. Sepsis and the systemic inflammatory response syndrome: neuromuscular manifestations. Crit Care Med 1996;24:1408-16.

2 Vukmir RB. Pyogenic hepatic abscess. Ann Emerg Med 1991;20:421-3.

3 Sridharan GV, Wilkinson SP, Primrose WR. Pyogenic liver abscess in the elderly. Age Ageing 1990;19:199-203.
Keywords: peripheral neuropathy; hepatic abscess

4 Young AE. The clinical presentation of pyogenic liver abscess. Br F Surg 1976;63:216-9.

5 Teitz S, Guidetti-Sharon A, Manor H, Halevy A. Pyogenic liver abscess: warning indicator of silent colonic cancer. Dis Colon Rectum 1995;38:1220-3.

\title{
Hypercalcaemia with radiographic abnormalities in chronic myeloid leukaemia
}

\author{
N Sharma, S Jain, S Kumari, S Varma
}

A 56-year-old woman with untreated chronic myeloid leukaemia presented with a history of weakness, easy fatigability and fever of 6 weeks duration. She was emaciated, pale, had sternal tenderness and a few palpable lymph nodes in the cervical, axillary and the inguinal areas. General physical examination showed splenomegaly $(2 \mathrm{~cm}$ below the left costal margin). Laboratory investigations revealed a haemoglobin level of $8.5 \mathrm{~g} / \mathrm{dl}$; a total leucocyte count of $125 \times 10^{9} / 1$ with $35 \%$ neutrophils, $4 \%$ lymphocytes, $1 \%$ monocytes, $37 \%$ eosinophils, $2 \%$ basophils, $1 \%$ promyelocytes, $10 \%$ myelocytes, $6 \%$ metamyelocytes, and $4 \%$ blasts, and there were $3-4$ nucleated red blood cells per 100 white blood cells. The peripheral blood smear showed microcytic and elliptocytic red blood cells. The platelet count was $200 \times 10^{9} / 1$. The serum biochemistry showed a serum calcium of $3.6 \mathrm{mmol} / \mathrm{l}$, serum albumin $26 \mathrm{~g} / \mathrm{l}$, serum phosphorous $1.3 \mathrm{mmol} / \mathrm{l}$ and serum alkaline phosphatase 241.4 IU/1 (normal 21.3-92.3 IU/1). Estimation of the 24-hour urine sample showed a calcium excretion of $660 \mathrm{mg}$ (normal $100-300 \mathrm{mg}$ ) and a phosphorous excretion of $1580 \mathrm{mg}$ (normal 600-1200 mg). The bone marrow of this patient was hypercellular with depressed erythropoiesis and a differential count of $12 \%$ blasts, $2 \%$ promyelocytes, $2 \%$ myelocytes, $3 \%$ metamyelocytes, $44 \%$ neutrophils, $3 \%$ lymphocytes, $2 \%$ monocytes and $32 \%$ eosinophils. The leucocyte alkaline phosphatase score was 42 (normal 35-140) and the trephine biopsy disclosed coarse deposition of reticulin. Photographs of the X-rays and a Technetium ${ }^{99 m}$ bone scan images are displayed in figures 1 and 2, respectively.

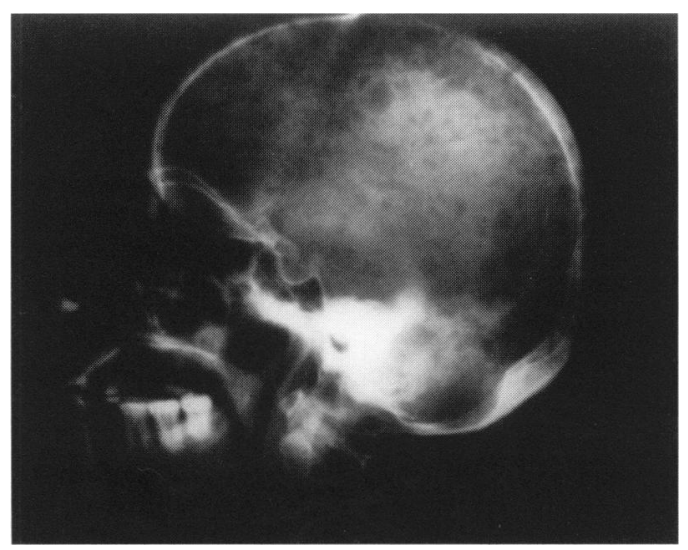

Figure 1 X-ray of the skull, lateral view

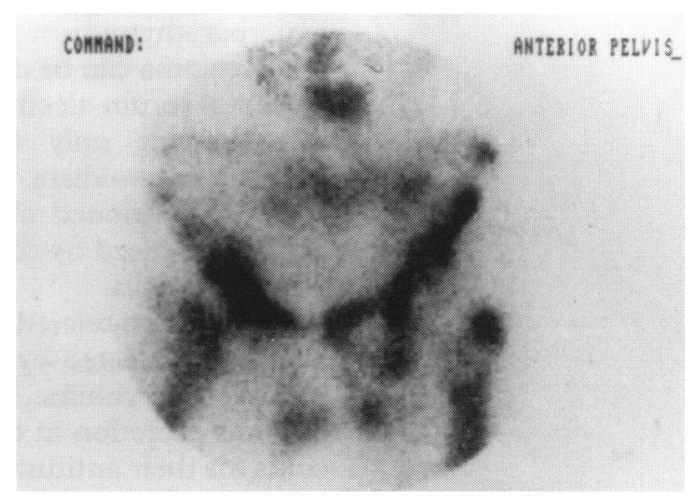

Figure 2 Technetium ${ }^{99 m}$ bone scan of the pelvis
Institute of Medical

Education and

Research, Chandigarh, India 160012

N Sharma

S Jain

S Kumari

$S$ Varma

Correspondence to $\mathrm{Dr}$ Sanjay Jain

Accepted 25 November 1997

\section{Questions}

1 What do the haematological parameters and the bone marrow findings of this patient indicate?

2 What does the serum biochemistry and analysis of the 24-hour urinary sample disclose? List the different conditions that can cause this state.

3 What do the $\mathrm{X}$-ray and the radionuclide scan depict? List the conditions that can manifest with such a picture and give their appropriate treatments. 